\title{
S100A4 interacts with mutant p53 and affects gastric cancer MKN1 cell autophagy and differentiation
}

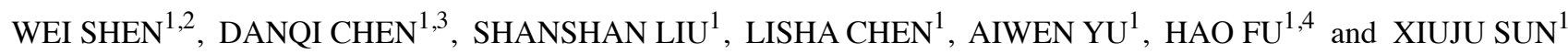 \\ ${ }^{1}$ Department of Medical Genetics, China Medical University, Shenyang North New Area, Shenyang, Liaoning 110122; \\ ${ }^{2}$ Department of Pathophysiology, Shenyang Medical College, Shenyang, Liaoning 110034, P.R. China; \\ ${ }^{3}$ Department of Environmental Medicine, NYU School of Medicine, Tuxedo, NY 10987, USA; \\ ${ }^{4}$ Department of Biochemistry, Shenyang Medical College, Shenyang, Liaoning 110034, P.R. China
}

Received June 10,2015; Accepted August 19, 2015

DOI: $10.3892 /$ ijo.2015.3209

\begin{abstract}
The acquired p53 mutations are the most common genetic alterations in human cancers. Mutant p53 proteins tend to accumulate, augmenting their oncogenic potential. However, the mechanisms for mutant p53 accumulation are not known. Previous studies have shown that S100A4 interacts with wild-type p53. The present study marks the first time the effect of S100A4 on mutant p53 levels in gastric cancer MKN1 cells, which harbor mutant $\mathrm{p} 53^{\mathrm{V} 143 \mathrm{~A}}$, and the functional consequences have been investigated. S100A4 interacted with mutant $\mathrm{p} 53^{\mathrm{V} 143 \mathrm{~A}}$ in the cells, and S100A4 inhibition decreased mutant $\mathrm{p} 53^{\mathrm{V} 143 \mathrm{~A}}$ levels, indicating that $\mathrm{S} 100 \mathrm{~A} 4$ promoted mutant p53 accumulation through their interaction. We also found that S100A4 inhibition altered the expression of the mutant p53 ${ }^{\mathrm{V} 143 \mathrm{~A}}$ target genes $[c-M y c$ and inhibitor of DNA binding 2 (Id2)]. Moreover, we demonstrated that S100A4 knockdown increased mutant p53-related autophagy and cell differentiation. In conclusion, our data suggest a novel mechanism for mutant $\mathrm{p} 53^{\mathrm{V} 143 \mathrm{~A}}$ accumulation and add a new facet to the role of S100A4 in cancer.
\end{abstract}

\section{Introduction}

The tumor suppressor gene TP53 plays a pivotal role in maintaining and regulating normal cellular functions. The acquired TP53 mutations are the most common genetic alterations in human cancer, which are mostly missense mutations (1). Some p53 mutants not only result in the loss of wild-type p53 activity, but also may acquire new oncogenic properties known as gain-of-function. The mutation usually leads to the formation of mutant p53 proteins, which often accumulate at high levels in tumors (2-4). In fact, immunohistochemical detection of p53 in tumors usually indicates TP53 missense

Correspondence to: Dr Xiuju Sun, Department of Medical Genetics, China Medical University, No. 77 Puhe Road, Shenyang North New Area, Shenyang, Liaoning 110122, P.R. China

E-mail:xjsun@mail.cmu.edu.cn

Key words: S100A4, mutant p53, protein-protein interaction, gastric cancer cell, autophagy mutation and provides prognostic and predictive information. As the notion that mutant p53 accumulation augments their oncogenic potential, the field of molecular modulators of mutant p53 level and activity is gaining interest. A prominent notion underlying mutant p53 accumulation is that the mutations in TP53 abrogate its ability to transactivate $M D M 2$, which typically regulates the ubiquitin-mediated degradation of p53 $(5,6)$. However, recent in vivo data challenge this hypothesis by demonstrating that mutant Trp53 knock in mice (Trp53 encodes mouse p53) do not have mutant p53 accumulation in normal tissues, whereas mutant p53 levels are increased in most tumors (7). The findings indicated that TP53 mutations alone are insufficient for the accumulation of mutant p53 and that additional events are required to prevent mutant p53 degradation. It has been reported that molecules such as heat shock proteins (HSP90) or phosphatase and tensin homolog (PTEN) interact with mutant p53 and lead to its accumulation in tumors $(8,9)$.

S100A4 (also known as Mts1) belongs to the S100 family of $\mathrm{Ca}^{2+}$-binding proteins that are overexpressed in a multitude of cancers and that are accompanied by increased metastatic capacity (10-12). S100A4 is involved in cancer progression and metastasis through interaction with target proteins. Using in vitro or in vivo binding assays, several groups have demonstrated the interaction between wild-type p53 and S100A4, and the functional consequence of wild-type p53-S100A4 interaction has been reported. Mutant p53 and S100A4 interaction in mouse mammary cancer CSML-100 cells has also been reported (13). However, whether mutant p53 and S100A4 can interact in human cancer cells and the functional consequences have yet to be revealed. The human gastric cancer cell line MKN1 harbors mutant $\mathrm{p} 53^{\mathrm{V} 143 \mathrm{~A}}$ (14). We investigated whether S100A4 and mutant p53 interact in MKN1 cells and whether the interaction contributes to the accumulation of mutant p53. We also studied the role of S100A4 in regulating mutant p53-related molecular and cellular effects.

\section{Materials and methods}

Cell culture. The human gastric cancer cell line MKN1 was kindly provided by professor Kazunari K. Yokoyama (Riken, The Institute of Physical and Chemical Research, Saitama, 
Japan). Cells were cultured in RPMI-1640 medium (Invitrogen, Carlsbad, CA, USA) supplemented with $10 \%$ fetal calf serum in a $37^{\circ} \mathrm{C}$ humidified incubator with $5 \% \mathrm{CO}_{2}$.

Double immunofluorescence. MKN1 cells were seeded on glass coverslips ( $1 \times 10^{5}$ cells $\left./ \mathrm{ml}\right)$. After $24 \mathrm{~h}$, the cells were fixed with methyl alcohol and acetone, and then blocked with $10 \%$ normal goat serum. The cell monolayer was treated overnight at $4^{\circ} \mathrm{C}$ with primary antibodies: rabbit anti-S100A4 antibody (1:100 dilution; Lab Vision, Fremont, CA USA) and mouse anti-p53 antibody (DO-1; Santa Cruz Biotechnology, Santa Cruz, CA, USA; 1:100 dilution). The p53 antibody (DO-1) is a mouse monoclonal antibody raised against amino acids 11-25 of p53 of human origin, which was recommended for detection of wild and mutant p53 of human origin according to the instruction book. In the present study, we used DO-1 to detect mutant p53 $^{\mathrm{V} 143 \mathrm{~A}}$ expression in MKN-1 cells. After incubating in primary antibodies, the cells were treated with fluorescein isothiocyanate-conjugated secondary goat anti-rabbit IgG and tetramethylrhodamine isothiocyanate-conjugated goat antimouse IgG (Sigma, St. Louis, MO, USA). Lastly, the nuclei were stained with diaminophenylindole (DAPI) in phosphatebuffered saline (PBS) for $10 \mathrm{~min}$. Specimens were examined under a Leica TCS SP2 AOBS confocal laser microscope (Leica Microsystems, Wetzlar, Germany).

Co-immunoprecipitation (Co-IP). Cells were washed with PBS and lysed in Triton lysis buffer. Cell extracts were precleared by incubating with $50 \mu \mathrm{l}$ Protein G PLUS-Agarose (Santa Cruz Biotechnology) for $30 \mathrm{~min}$ at $4^{\circ} \mathrm{C}$ and centrifuged. Precleared cell extracts were incubated with $4 \mu \mathrm{g}$ mouse anti-p53 antibody or rabbit anti-S100A4 antibody at $4^{\circ} \mathrm{C}$ for $1 \mathrm{~h}$. IgG was used as the control for the Co-IP. Thereafter, $70 \mu 1$ resuspended Protein G PLUS-Agarose was added to the lysate-antibody mix and incubated overnight at $4^{\circ} \mathrm{C}$ on a rotating wheel. After incubation, the beads were centrifuged and the supernatant was discarded. The beads were washed four times with PBS, resuspended and boiled in $20 \mu \mathrm{l}$ SDS sample buffer, and immunoblotted with anti-S100A4 antibody or anti-p53 antibody (1:200 dilution).

Plasmid construction and cell transient transfection. The pCMV-Neo-Bam $5^{\text {V143A }}$ expression vector was a gift from Professor Bert Vogelstein (Johns Hopkins University, Baltimore, MD, USA). The S100A4-specific shRNA expression vector was constructed in a previous study by our group (15). The double-stranded shRNA oligo was cloned into a pSilencer 4.1-CMV neo vector (Ambion, Austin, TX, USA) to construct pS100A4-shRNA. A scrambled sequence without significant homology to human gene sequences was used as the control (pControl-shRNA). Cell transfections were carried out using Lipofectamine 2000 (Invitrogen) according to the manufacturer's instruction.

RNA extraction and semi-quantitative RT-PCR. Total cellular RNA was extracted using TRIzol reagent (Invitrogen); $1 \mu \mathrm{g}$ RNA was reverse-transcribed using First-Strand cDNA Synthesis kit (Promega, Madison, WI, USA). The newly synthesized complementary DNA was amplified by PCR. Primers specific for human TP53, S100A4 and $\beta$-actin were designed as follows: (TP53) sense, 5'-CAGCCAAGTCTGTG ACTTGCACGTAC-3' and antisense, 5'-CTATGTCGAAAAG TGTTTCTGTCATC-3'; (S100A4) sense, 5'-GATGTGATGG TGTCCACCTT-3' and antisense, 5'-ATTTCTTCCTGGG CTGCTTA-3'; ( $\beta$-actin) sense, 5'-CTCTTCCAGCCTTCCTT CCT-3' and antisense, 5'-CACCTTCACCGTTCCAGTTT-3'. Amplification cycles were: $95^{\circ} \mathrm{C}$ for $5 \mathrm{~min}$, and then 30 cycles at $95^{\circ} \mathrm{C}$ for $30 \mathrm{sec}, 58^{\circ} \mathrm{C}$ for $30 \mathrm{sec}$, and $72^{\circ} \mathrm{C}$ for $30 \mathrm{sec}$, followed by $72^{\circ} \mathrm{C}$ for $10 \mathrm{~min}$. Aliquots of the PCR product were electrophoresed on $1.5 \%$ agarose gels, and PCR fragments were visualized by ethidium bromide staining.

Quantitative real-time RT-PCR. The RNA extraction and reverse transcription reaction were performed as described above. Quantitative real-time PCR analysis was performed by using SYBR Premix Ex Taq II (Takara Bio, Dalian, China). Reactions were processed and analyzed on an ABI 7500 Real-Time PCR system (Applied Biosystems, Foster City, CA, USA). The sense and antisense primers used for $I d 2, c-M y c$, and glyceraldehyde-3-phosphate dehydrogenase $(G A P D H)$ were 5'-TCAGCCTGCATCACCAGAGA-3' and 5'-CTGC AAGGACAGGATGCTGATA-3'; 5'-ACCAGATCCCGGAG TTGGAA-3' and 5'-CGTCGTTTCCGCAACAAGTC-3'; and 5'-ATCATCAGCAATGCCTCC-3' and 5'-CATCACGCCAC AGTTTC-3', respectively.

Western blotting. Whole cell extracts were prepared from cells by homogenizing cells in a lysis buffer (50 mM Tris, $\mathrm{pH} 7.2,1 \%$ Triton X-100, $0.5 \%$ sodium deoxycholate, $0.1 \%$ SDS, $500 \mathrm{mM}$ $\mathrm{NaCl}, 10 \mathrm{mM} \mathrm{MgCl}$, with $10 \mu \mathrm{g} / \mathrm{ml}$ leupeptin, $10 \mu \mathrm{g} / \mathrm{ml}$ aprotinin and $1 \mathrm{mM}$ PMSF) and quantified by Bradford method. Protein $(100 \mu \mathrm{g})$ was separated on a $12 \%$ polyacrylamide gel by electrophoresis, transferred onto polyvinylidene difluoride membranes (Millipore, Bedford, MA, USA) and blocked with TBS-T supplemented with 5\% non-fat milk. Membranes were incubated with rabbit anti-S100A4 antibody (1:500 dilution) and mouse anti-p53 antibody (1:500 dilution), rabbit anti$\beta$-actin antibody (1:1,000 dilution; Santa Cruz Biotechnology). After washing, membranes were incubated with a peroxidaseconjugated second antibody (goat anti-rabbit IgG or goat anti-mouse IgG) (1:2,000 dilution; Beijing Zhongshan Golden Bridge Biotechnology Co., Ltd., Beijing, China). The reagent for enhanced chemiluminescence (Amersham Biosciences, Freiburg, Germany) was used for detection and developed by $\mathrm{X}$-ray film. The experiments were performed three times.

Chromatin immunoprecipitation assays (ChIP). ChIP assays were performed according to the manufacturer's instructions (Active Motif, Carlsbad, CA, USA). The target protein p53 was immunoprecipitated with either $3 \mu \mathrm{g}$ anti-p53 antibody or $3 \mu \mathrm{g} \mathrm{IgG}$ as the negative control. DNA was extracted as recommended by the protocol. To amplify the mutant p53 binding site from nucleotides -163 to +22 in the $I d 2$ gene promoter region, PCR was performed using the forward and reverse primers 5'-GCACTTACTGTACTGTACTCTAT-3' and 5'-GCTGGAGCTTCCCTTCGTC-3', respectively (16).

Induction and quantification of autophagy. To analyze autophagosomes, we constructed pEGFP-C1-LC3 expression vectors and transfected them into MKN1 cells five days after 

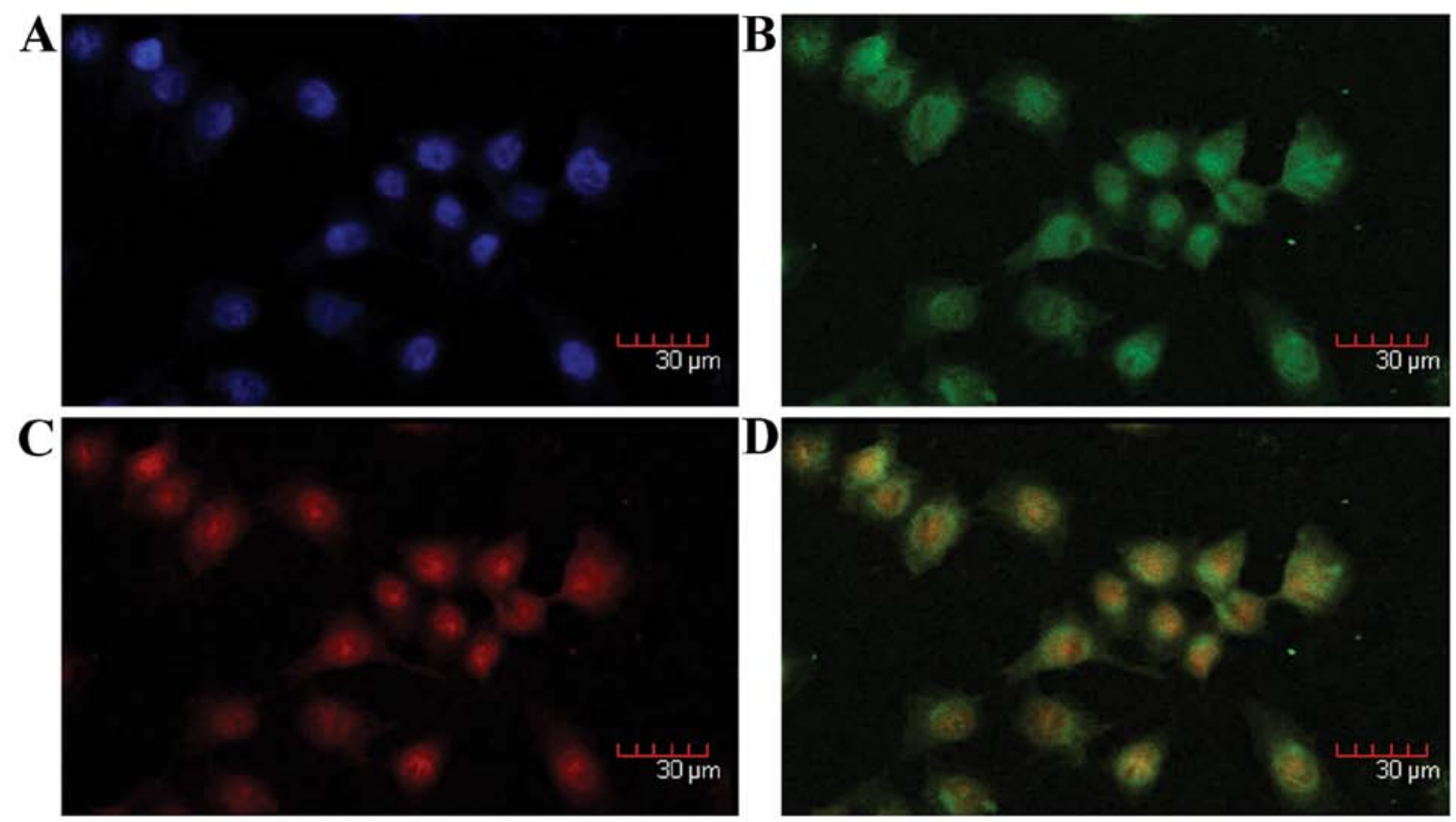

Figure 1. S100A4 and mutant p53 $3^{\mathrm{V} 143 \mathrm{~A}}$ colocalization in MKN1 cells under confocal microscopy. (A) DAPI nuclear staining. (B and C) Expression of S100A4 and mutant $\mathrm{p} 53^{\mathrm{V} 143 \mathrm{~A}}$, respectively. (D) Colocalization of S100A4 (green) and mutant $\mathrm{p}^{\mathrm{V}} 3^{\mathrm{V} 143 \mathrm{~A}}$ (red).

pS100A4-shRNA or pControl-shRNA transfection in the cells. After $36 \mathrm{~h}$, cells were cultured for $12 \mathrm{~h}$ in serum-free Hank's balanced salt solution medium, referred to as nutrientfree medium, for serum and amino acid starvation (17). Autophagy was quantified by determining the percentage of cells with GFP-LC3 accumulation in vacuoles (GFP-LC3vac, of a minimum 100 cells per preparation in three independent experiments). Under fluorescence microscopy, cells presenting mostly diffuse distribution of GFP-LC3 in the cytoplasm and nucleus were considered non-autophagic, whereas cells with several intense punctate GFP-LC3 aggregates with no nuclear GFP-LC3 were classified as autophagic (18). Each GFP-LC3 staining was read by two independent investigators.

Measurement of ALP activity. MKN1 cells were harvested by trypsinization and rinsed twice with PBS five days after pS100A4-shRNA or pControl-shRNA transfection. Then, cells were incubated in $0.2 \%$ Triton $\mathrm{X}-100$ for $24 \mathrm{~h}$ at $4^{\circ} \mathrm{C}$, and sonicated. ALP activities in the cell lysates were measured by determining the formation of $p$-nitrophenol from $p$-nitrophenol phosphate using a commercially available kit (Nanjing Jiancheng Biotech Co., Ltd., Nanjing, China) according to the manufacturer's instructions. ALP activity was expressed as ALP units per mg protein. All results were normalized by protein quantitation. The results were repeated in at least three independent experiments.

Statistical analysis. The data are presented as mean \pm SD (standard deviation). Each experiment was repeated at least three times. Statistical analyses were performed using the Student's t-test or analysis of variance (ANOVA) according to the number of groups compared. Student-Newman-Keuls (SNK) test was used after the ANOVA for pairwise comparison. All analyses used SPSS version 16.0 software. A P-value of $<0.05$ was considered statistically significant.

\section{Results}

S100A4 and mutant p53 $3^{V 143 A}$ colocalization in MKN1 cells. The colocalization of S100A4 and mutant $\mathrm{p} 53^{\mathrm{V} 143 \mathrm{~A}}$ in the nucleus and cytoplasm of MKN1 cells was identified by double immunofluorescence staining (Fig. 1).

S100A4 interaction with mutant $p 53^{\text {VI43A }}$ in MKN1 cells. As S100A4 and mutant $\mathrm{p} 53^{\mathrm{V} 143 \mathrm{~A}}$ were colocalized in MKN1 cells, we hypothesized that S100A4 could interact with the mutant p53 in the cells. To investigate the interaction, whole cell lysates of MKN1 cells were prepared and precipitated with anti-S100A4 antibody, and the proteins from the immunoprecipitates were detected by western blotting using anti-p53 antibody. We detected mutant $\mathrm{p} 53^{\mathrm{V} 143 \mathrm{~A}}$, which indicated that it coprecipitated with S100A4. Similarly, when whole cell lysates were precipitated with anti-p53 antibody, S100A4 was detected from the immunoprecipitates (Fig. 2), indicating that S100A4 can coprecipitate with mutant $\mathrm{p} 53^{\mathrm{V} 143 \mathrm{~A}}$. Normal IgG was used as the negative control. These results demonstrate that S100A4 interacts with mutant $\mathrm{p} 53^{\mathrm{V} 143 \mathrm{~A}}$ in MKN1 cells.

Effect of S100A4 on mutant p53 ${ }^{\text {VI43A }}$ levels. After demonstrating that S100A4 could interact with mutant $\mathrm{p} 53^{\mathrm{V} 143 \mathrm{~A}}$, we used western blotting and RNA interference to investigate whether inhibiting S100A4 would affect mutant p53 levels. S100A4 inhibition (Fig. 3A and B) decreased mutant p53 $3^{\mathrm{V} 143 \mathrm{~A}}$ levels (Fig. 3C), indicating that $\mathrm{S} 100 \mathrm{~A} 4$ could contribute to the accumulation of mutant $\mathrm{p} 53^{\mathrm{V} 143 \mathrm{~A}}$ in $\mathrm{MKN} 1$ cells. 
A

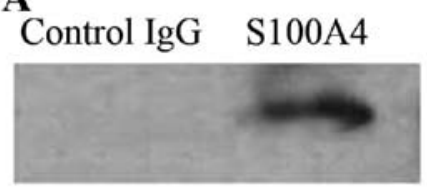

B

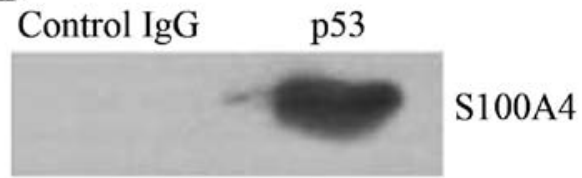

Figure 2. Co-IP analysis of S100A4 and mutant p53 $3^{\mathrm{V} 143 \mathrm{~A}}$ interaction in MKN1 cells. (A) Cell extracts were immunoprecipitated with anti-S100A4 antibody and immunoblotted for mutant p53. (B) Cell extracts were immunoprecipitated with anti-p53 antibody and immunoblotted for S100A4.

$\mathbf{A}$
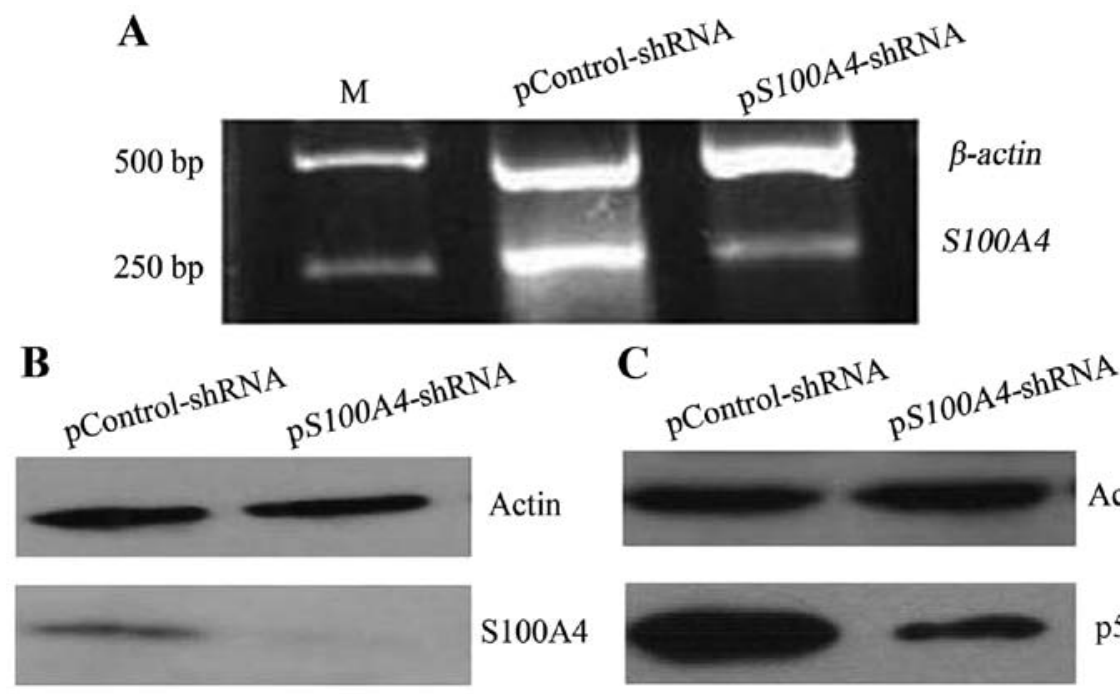

S100A4
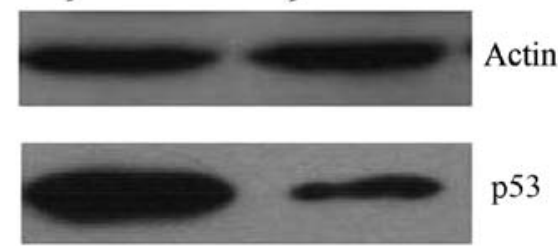

Figure 3. Effect of S100A4 on mutant p53 ${ }^{\mathrm{V} 143 \mathrm{~A}}$ levels. (A) RT-PCR or (B) western blotting measurement of S100A4 mRNA and protein levels in pS100A4shRNA or pControl-shRNA cells. Lane M, DNA marker DL2000. (C) Western blotting measurement of p53 levels in pS100A4-shRNA or pControl-shRNA cells.

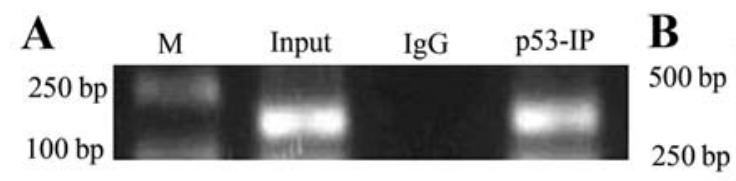

C
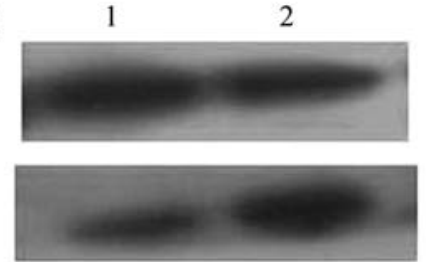

Actin

p53
D
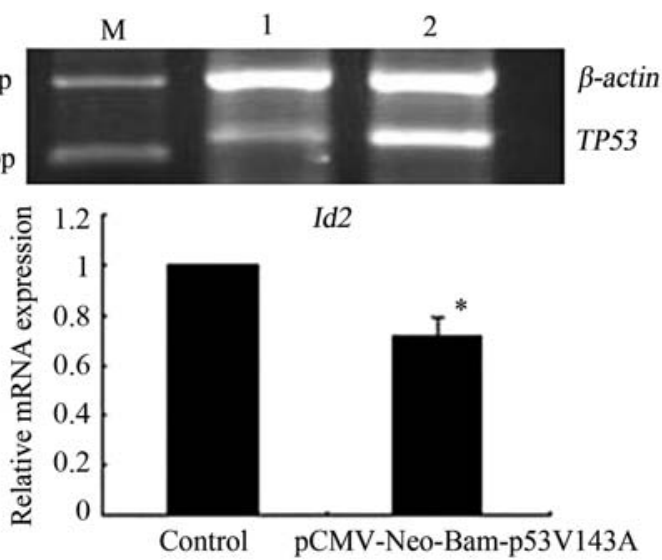

Figure 4. Identification of $I d 2$ as a new target gene of mutant $T P 53^{V 143 A}$. (A) ChIP detected the binding of p 53 to the $I d 2$ promoter in MKN1 cells. (B) RT-PCR or western blotting (C) measurement of TP53 mRNA and protein expression after pCMV-Neo-Bam p53 ${ }^{\mathrm{V} 143 \mathrm{~A}}$ transfection. Lane M, DNA marker DL2000; lane 1, untransfected control cell; lane 2, cell transfected with pCMV-Neo-Bam p53 ${ }^{\mathrm{V} 143 \mathrm{~A}}$. (D) $I d 2 \mathrm{mRNA}$ expression after pCMV-Neo-Bam p53 $3^{\mathrm{V} 143 \mathrm{~A}}$ transfection. The data represent the mean $\pm \mathrm{SD}$ from three experiments $\left({ }^{*} \mathrm{P}<0.05\right)$.

Inhibitor of DNA binding 2 (Id2) was a target gene of mutant $T P 53^{V 143 A} . I d 2$ is a target gene of some p53 mutants, such as $\mathrm{p} 53^{\mathrm{R} 273 \mathrm{H}}, \mathrm{p} 53^{\mathrm{P} 309 \mathrm{~S}}$ and $\mathrm{p} 53^{\mathrm{R} 248 \mathrm{~W}}(16)$, but it is not clear whether it is the target gene of mutant $T P 53^{V 143 A}$. To investigate this, we first performed chromatin immunoprecipitation (ChIP), and the results showed that mutant $T P 53^{V I 43 A}$ bound to the promoter region of the $I d 2$ gene (Fig. 4A). We further investigated the effect of $T P 53^{V I 43 A}$ on Id 2 expression via pCMV-Neo-Bam p53 $3^{\mathrm{V} 143 \mathrm{~A}}$ transfection. RT-PCR and western blotting showed that the levels of TP53 mRNA and protein expression were significantly increased at $48 \mathrm{~h}$ after pCMV-Neo-Bam p53 ${ }^{\mathrm{V} 143 \mathrm{~A}}$ transfection compared to the control cells (Fig. 4B and C), indicating that the pCMV-Neo-Bam $\mathrm{p}^{\mathrm{V}}{ }^{\mathrm{V} 143 \mathrm{~A}}$ transfection led to mutant $T P 53^{V I 43 A}$ overexpression in the cells. Mutant $T P 53^{V 143 A}$ overexpression decreased Id 2 mRNA expression in the cells (Fig. 4D). The results indicate that $I d 2$ is a target gene 
A

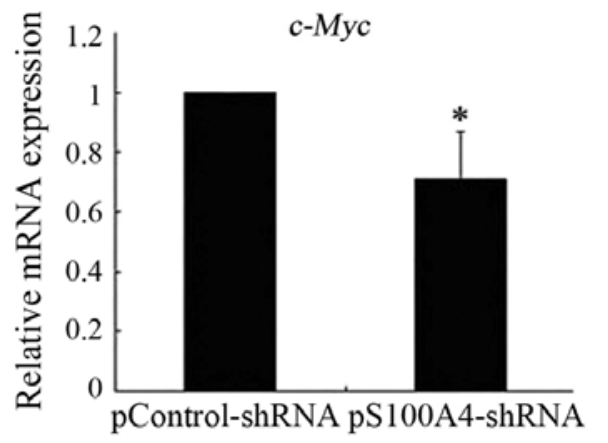

B

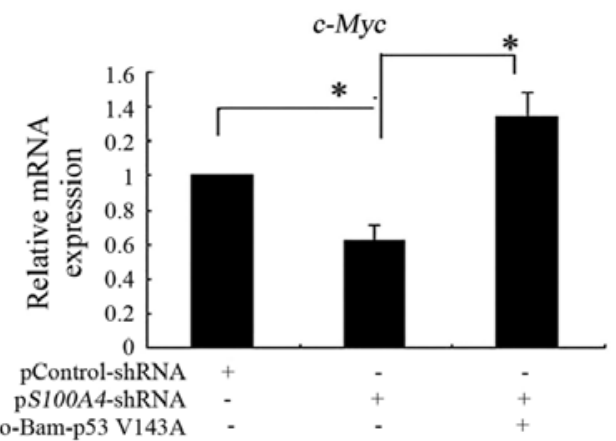

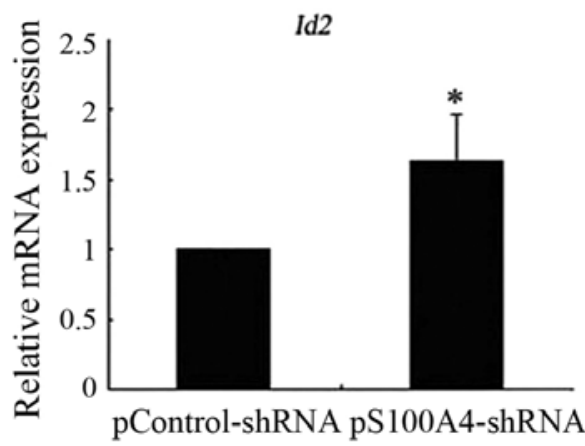

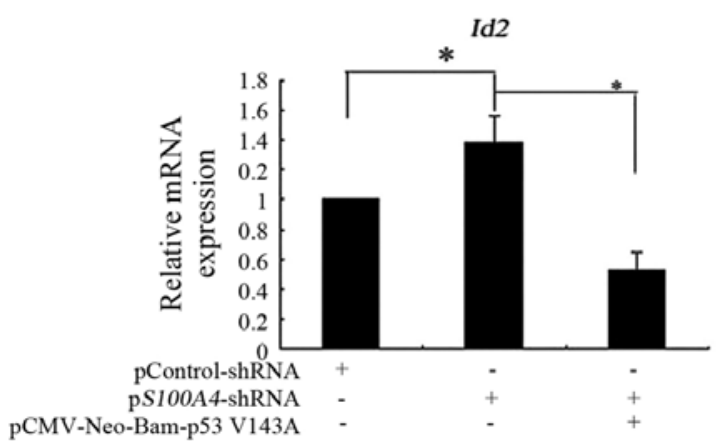

Figure 5. S100A4 affects the expression of mutant TP53 ${ }^{V 143 A}$ target genes. (A) Real-time PCR detection of $c$-Myc or Id2 mRNA expression after S100A4 inhibition. Data represent the mean $\pm \mathrm{SD}(\mathrm{n}=3),{ }^{*} \mathrm{P}<0.05$. (B) Real-time RT-PCR detection of $c$-Myc or Id2 mRNA expression after $S 100 A 4-$ shRNA and pCMV-Neo-Bam $553^{\mathrm{V} 143 \mathrm{~A}}$ cotransfection. Data represent the mean $\pm \mathrm{SD}(\mathrm{n}=3),{ }^{*} \mathrm{P}<0.05$.

of mutant $T P 53^{V 143 A}$ and that its expression is directly repressed by mutant $T P 53^{V 143 A}$.

S100A4 affected the expression of mutant TP53 ${ }^{\text {V143A }}$ target genes. It has been reported that $c-M y c$ is a target gene of mutant $\mathrm{p} 53^{\mathrm{V} 143 \mathrm{~A}}(19)$; our results above indicate that $I d 2$ is a target gene of mutant TP53 $3^{V 143 A}$. Therefore, we performed real-time RT-PCR to investigate the effect of S100A4 on $c-M y c$ and Id 2 mRNA expression. S100A4 inhibition decreased the expression of $c-M y c$ mRNA and increased the expression of Id2 mRNA (Fig. 5A). Rescue experiments showed that pCMVNeo-Bam p53 ${ }^{\mathrm{V} 143 \mathrm{~A}}$ transfection into pS100A4-short hairpin RNA (shRNA) cells attenuated the decreased expression of $c-M y c$ mRNA and the increased expression of Id 2 mRNA induced by S100A4 suppression (Fig. 5B). Collectively, the results suggest that $\mathrm{S} 100 \mathrm{~A} 4$ affects the expression of mutant $T P 53^{V I 43 A}$ target genes such as $c-M y c$ and $I d 2$, therefore, it affects the molecular function of mutant TP53 ${ }^{V 143 A}$.

S100A4 suppression promoted MKN1 cell autophagy and differentiation. We demonstrated that S100A4 interacts with mutant p53 and affects mutant p53 levels. It has been reported that there is a good correlation between many p53 variants (including $\mathrm{p} 53^{\mathrm{V} 143 \mathrm{~A}}$ ) and their ability to inhibit autophagy (20). It presents the possibility that S100A4 may affect MKN1 cell autophagy through mutant $\mathrm{p} 53^{\mathrm{V} 143 \mathrm{~A}}$. Therefore, we examined the potential effect of S100A4 in inducing autophagy. Knockdown of S100A4 significantly increased green fluorescent protein-light chain 3 (GFP-LC3) aggregation in the cytoplasmic dots induced by nutrient deprivation (Fig. 6A), indicating that S100A4 suppression could increase MKN1 cell autophagy.
We determined that S100A4 affected the expression of the mutant TP53 $3^{V I 43 A}$ target genes $I d 2$ and $c-M y c$. The $I d 2$ and $c-M y c$ genes are implicated in the regulation of cell differentiation $(16,21)$. We hypothesized that S100A4 may affect cell differentiation. Alkaline phosphatase (ALP) activity is a marker of differentiation. The expression of an ALP isoenzyme was stronger in well-differentiated gastric carcinoma than in poorly differentiated carcinoma (22). In the present study, ALP activity was significantly increased in pS100A4shRNA cells compared to in pControl-shRNA cells (Fig. 6B), indicating that S100A4 inhibition may promote MKN1 cell differentiation.

\section{Discussion}

Using in vitro binding studies, several different groups have demonstrated direct interaction between recombinant p53 and S100A4 using different methods (23-25). A report has also been published on the interaction between S100A4 and p53 in a complex sample involving coimmunoprecipitation (Co-IP) of mouse mammary cancer cells harboring mutant p53 (13). Recent research has shown that S100A4 interacted with wildtype p53 in human cancer cells (26). However, whether S100A4 and mutant p53 interact in human cancer cells, and whether S100A4 affects mutant p53 accumulation is not clear. The present study marks the first report of the nuclear and cytoplasmic colocalization of S100A4 and mutant p53, as well as the in vivo interaction between S100A4 and mutant p53, which was detected using double immunofluorescence and Co-IP in MKN1 cells, which harbor mutant p53 ${ }^{\mathrm{V} 143 \mathrm{~A}}$. Importantly, our results show that $\mathrm{S100A} 4$ inhibition decreased mutant $\mathrm{p} 53^{\mathrm{V} 143 \mathrm{~A}}$ expression, which suggests that S100A4 might promote mutant 


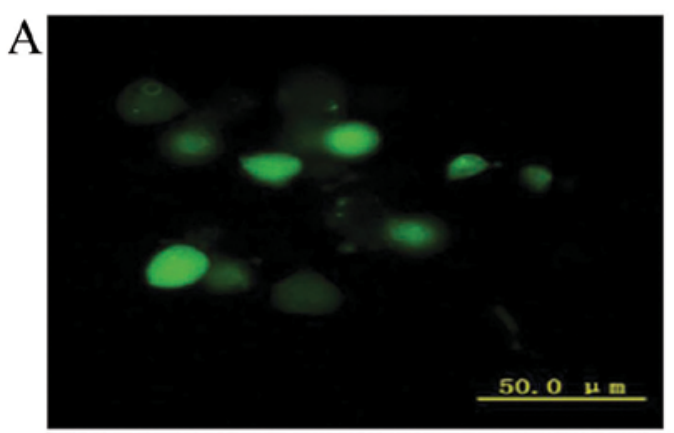

pControl-shRNA (nutrient-free)

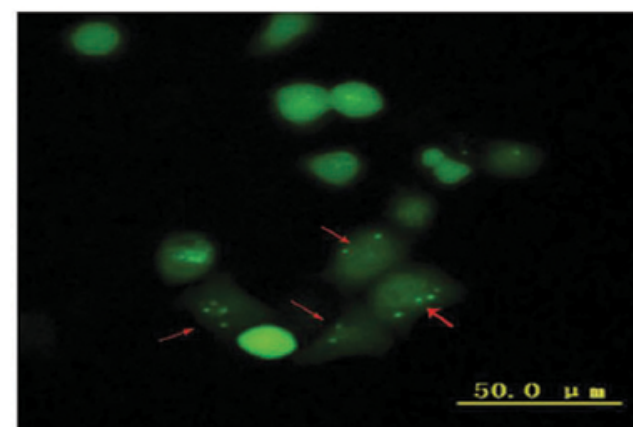

pS100A4-shRNA (nutrient-free)
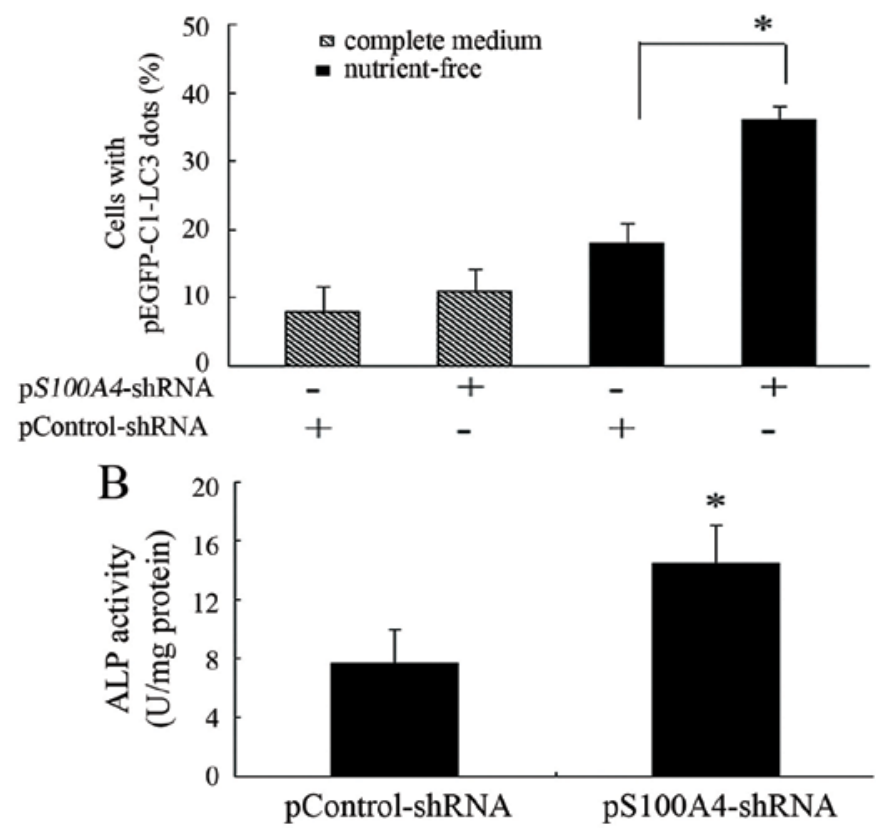

Figure 6. Effects of S100A4 on MKN1 cell autophagy and differentiation. (A) Effect of S100A4 inhibition on MKN1 cell autophagy. Image depicts fluorescence microscopy detection of GFP-LC3 aggregation in the cells. Shown are representative fluorescence microphotographs and quantitative results (mean \pm SD, $\mathrm{n}=3$ ), (B) Effect of S100A4 inhibition on MKN1 cell differentiation. ALP activity was plotted as mean from triplicate experiments. Bars indicate SD; ${ }^{*} \mathrm{P}<0.05$ vs. the pControl-shRNA cells.

p53 ${ }^{\mathrm{V} 143 \mathrm{~A}}$ accumulation. The mechanism may depend on the interaction between S100A4 and mutant $\mathrm{p} 53^{\mathrm{V} 143 \mathrm{~A}}$. A recent report demonstrated the frequent combination of mutated TP53-positive and S100A4-positive status in colorectal carcinoma samples (27), which supports our findings from a clinicopathological perspective. As described above, it was reported recently that endogenous S100A4 and wild-type p53 interact in complex samples, and S100A4 knockdown resulted in p53 stabilization in two wild-type p53 cell lines, indicating that S100A4 promotes wild-type p53 degradation (26). These reports suggested that S100A4 has the opposite effect on wildtype p53 compared to its effect on mutant p53, as reported in this study. Similar to the effect of S100A4 on p53, the molecular chaperone HSP90, another partner of p53, also has the opposite effect on wild-type p53 compared to its effect on mutant p53. Wild-type p53 accumulated following HSP90 inhibition, whereas mutant p53 protein levels were reduced (9). These findings suggest that mutant and wild-type p53 might demonstrate different dependence on the same partner.

It is well known that p53 exerts its functions by regulating its target genes. Gain of function is dependent on the ability of mutant p53 to transactivate or repress specific target genes, such as $c-M y c, F a s$, and nuclear factor- $\kappa \mathrm{B}(\mathrm{NF}-\kappa \mathrm{B})(19,28,29)$. The genes affected by the different p53 mutants vary. Id 2 is a member of the inhibitor of differentiation (Id) family, which plays a role in tumor suppression in multiple tumor types. A previous study showed that $I d 2$ is a target gene of multiple p53 mutants, such as p53 ${ }^{\mathrm{R} 273 \mathrm{H}}, \mathrm{p} 53^{\mathrm{P} 309 \mathrm{~S}}$ and $\mathrm{p} 53^{\mathrm{R} 248 \mathrm{~W}}(16)$. The present study is the first to demonstrate that mutant $\mathrm{p} 53^{\mathrm{V} 143 \mathrm{~A}}$ inhibits $I d 2$ expression by binding to the $I d 2$ promoter, which suggests that $I d 2$ is a target gene of mutant $\mathrm{p} 53^{\mathrm{V} 143 \mathrm{~A}}$.

As S100A4 is frequently overexpressed in gastric cancer (11), and we found that S100A4 was responsible for mutant p53 accumulation by interacting with it in gastric cancer cells, we speculated that S100A4 could augment the oncogenic ability of mutant p53 in cells. How S100A4 affects mutant p53 activity requires further clarification. It has been reported that S100A4 binding to wild-type p53 interferes with the DNA binding activity of p53 in vitro and with reporter gene transactivation in vivo (in two mouse cancer cell lines: CSML-0 and VMR-Liv). In Tet-inducible cell lines expressing wild-type p53, differential modulation of the wild-type p53 target gene transcription $[P 21 / W A F, B A X$, thrombospondin-1 (THBS1), MDM2] was observed upon S100A4 induction (13). We 
hypothesized that S100A4 may affect the target gene expression of mutant $\mathrm{p} 53^{\mathrm{V} 143 \mathrm{~A}}$. We detected the effect of S100A4 on the expression of $c-M y c$ and $I d 2$, two target genes of mutant p53 ${ }^{\mathrm{V} 143 \mathrm{~A}}$. S100A4 knockdown decreased $c-M y c$ mRNA expression and increased Id 2 mRNA expression. Rescue assays showed that ectopic expression of mutant $\mathrm{p} 53^{\mathrm{V} 143 \mathrm{~A}}$ reversed the expression altered by S100A4 suppression. The above findings suggest that interaction with S100A4 altered the gene-specific regulation by mutant $\mathrm{p} 53 \mathrm{~V}^{143 \mathrm{~A}}$. Thus, our data add a new facet to the oncogenic properties of S100A4, where it affects mutant p53 target gene expression.

We further investigated the cellular consequences associated with mutant $\mathrm{p} 53$. In line with the gain-of-function hypothesis, mutant p53 destabilization upon HSP90 inhibition is accompanied by cell death (9). The authors also demonstrated that S100A4 knockdown led to wild-type p53-dependent cell cycle arrest and increased cisplatin-induced apoptosis (26). Autophagy is a catabolic process in which portions of the cytoplasmic organelles are sequestered within autophagosomes, and then targeted for bulk degradation. Disabled autophagy can accelerate tumor progression. It was reported that p53 mutants, including $\mathrm{p} 53 \mathrm{~V}^{143 \mathrm{~A}}$, effectively repress autophagy (20). We therefore hypothesized that, as a partner of mutant p53 ${ }^{\mathrm{V} 143 \mathrm{~A}}$, S100A4 could affect MKN1 cell autophagy, where S100A4 suppression increased it. These results mark the first time the effect of S100A4 on gastric cancer cell autophagy has been reported, and we hypothesize that mutant $\mathrm{p} 53^{\mathrm{V} 143 \mathrm{~A}}$ mediates this effect, although it remains to be proven.

We found that S100A4 affected the expression of the mutant TP53 ${ }^{V 143 A}$ target genes $I d 2$ and $c-M y c$, which regulate cell differentiation $(16,21)$. Clinical reports have shown that S100A4 overexpression is associated with poor differentiation in carcinoma (30). However, the role of S100A4 in gastric cancer cell differentiation remains unclear. In the present study, ALP activity showed that S100A4 inhibition might promote differentiation in gastric cancer MKN1 cells. We speculate that S100A4 may inhibit cell differentiation through mutant $\mathrm{p} 53^{\mathrm{V} 143 \mathrm{~A}}$ and its target genes such as $I d 2$ and $c-M y c$.

In conclusion, $\mathrm{S} 100 \mathrm{~A} 4$ interacts with mutant $\mathrm{p} 53^{\mathrm{V} 143 \mathrm{~A}}$, and is responsible for the accumulation of mutant $\mathrm{p} 53^{\mathrm{V} 143 \mathrm{~A}}$ in human gastric cancer MKN1 cells. Subsequently, S100A4 affects the expression of mutant $\mathrm{p} 53^{\mathrm{V} 143 \mathrm{~A}}$ target genes in the cells and further affects cellular characteristics such as autophagy and cell differentiation. S100A4 may be a powerful therapeutic target for inhibiting gain-of-function p53 mutants in gastric cancer.

\section{Acknowledgements}

We would like to thank Professor Bert Vogelstein for kindly providing the mutant $\mathrm{p} 53^{\mathrm{V} 143 \mathrm{~A}}$ constructs available to us. The gastric cancer cell line MKN1 was kindly provided by Professor Kazunari K. Yokoyama. This project was supported by grants from the National Natural Science Foundation of China (nos. 81272717 and 30570848) and the Liaoning Natural Science Foundation (no. 20102289).

\section{References}

1. Vogelstein B, Lane D and Levine AJ: Surfing the p53 network Nature 408: 307-310, 2000 .
2. Kastan MB and Berkovich E: p53: a two-faced cancer gene. Nat Cell Biol 9: 489-491, 2007.

3. Bossi G, Marampon F, Maor-Aloni R, Zani B, Rotter V, Oren M, Strano S, Blandino G and Sacchi A: Conditional RNA interference in vivo to study mutant p53 oncogenic gain of function on tumor malignancy. Cell Cycle 7: 1870-1879, 2008.

4. Adorno M, Cordenonsi M, Montagner M, Dupont S, Wong C, Hann B, Solari A, Bobisse S, Rondina MB, Guzzardo V, et al: A Mutant-p53/Smad complex opposes p63 to empower TGFbetainduced metastasis. Cell 137: 87-98, 2009.

5. Lukashchuk $\mathrm{N}$ and Vousden KH: Ubiquitination and degradation of mutant p53. Mol Cell Biol 27: 8284-8295, 2007.

6. Muller P, Hrstka R, Coomber D, Lane DP and Vojtesek B: Chaperone-dependent stabilization and degradation of $\mathrm{p} 53$ mutants. Oncogene 27: 3371-3383, 2008.

7. Olive KP, Tuveson DA, Ruhe ZC, Yin B, Willis NA, Bronson RT, Crowley D and Jacks T: Mutant p53 gain of function in two mouse models of Li-Fraumeni syndrome. Cell 119: 847-860, 2004.

8. Li Y, Guessous F, Kwon S, Kumar M, Ibidapo O, Fuller L, Johnson E, Lal B, Hussaini I, Bao Y, et al: PTEN has tumorpromoting properties in the setting of gain-of-function p53 mutations. Cancer Res 68: 1723-1731, 2008.

9. Lin K, Rockliffe N, Johnson GG, Sherrington PD and Pettitt AR: Hsp90 inhibition has opposing effects on wild-type and mutant p53 and induces p21 expression and cytotoxicity irrespective of p53/ATM status in chronic lymphocytic leukaemia cells. Oncogene 27: 2445-2455, 2008.

10. Zou M, Al-Baradie RS, Al-Hindi H, Farid NR and Shi Y: S100A4 (Mts1) gene overexpression is associated with invasion and metastasis of papillary thyroid carcinoma. Br J Cancer 93: 1277-1284, 2005

11. Cho YG, Nam SW, Kim TY, Kim YS, Kim CJ, Park JY, Lee JH, Kim HS, Lee JW, Park CH, et al: Overexpression of S100A4 is closely related to the aggressiveness of gastric cancer. APMIS 111: 539-545, 2003.

12. Matsumoto K, Irie A, Satoh T, Ishii J, Iwabuchi K, Iwamura M, Egawa S and Baba S: Expression of S100A2 and S100A4 predicts for disease progression and patient survival in bladder cancer. Urology 70: 602-607, 2007.

13. Grigorian M, Andresen S, Tulchinsky E, Kriajevska M, Carlberg C, Kruse C, Cohn M, Ambartsumian N, Christensen A, Selivanova $\mathrm{G}$, et al: Tumor suppressor $\mathrm{p} 53$ protein is a new target for the metastasis-associated Mts1/S100A4 protein: Functional consequences of their interaction. J Biol Chem 276: 22699-22708, 2001.

14. Yamada Y, Yoshida T, Hayashi K, Sekiya T, Yokota J, Hirohashi S, Nakatani K, Nakano H, Sugimura T and Terada M: p53 gene mutations in gastric cancer metastases and in gastric cancer cell lines derived from metastases. Cancer Res 51: 5800-5805, 1991.

15. Hua J, Chen D, Fu H, Zhang R, Shen W, Liu S, Sun K and Sun X: Short hairpin RNA-mediated inhibition of S100A4 promotes apoptosis and suppresses proliferation of BGC823 gastric cancer cells in vitro and in vivo. Cancer Lett 292: 41-47, 2010.

16. Yan W, Liu G, Scoumanne A and Chen X: Suppression of inhibitor of differentiation 2, a target of mutant p53, is required for gain-of-function mutations. Cancer Res 68: 6789-6796, 2008.

17. Mizushima N, Yamamoto A, Matsui M, Yoshimori T and Ohsumi Y: In vivo analysis of autophagy in response to nutrient starvation using transgenic mice expressing a fluorescent autophagosome marker. Mol Biol Cell 15: 1101-1111, 2004.

18. Kanzawa T, Zhang L, Xiao L, Germano IM, Kondo Y and Kondo S: Arsenic trioxide induces autophagic cell death in malignant glioma cells by upregulation of mitochondrial cell death protein BNIP3. Oncogene 24: 980-991, 2005.

19. Frazier MW, He X, Wang J, Gu Z, Cleveland JL and Zambetti GP: Activation of c-myc gene expression by tumor-derived p53 mutants requires a discrete C-terminal domain. Mol Cell Biol 18: 3735-3743, 1998.

20. Morselli E, Tasdemir E, Maiuri MC, Galluzzi L, Kepp O, Criollo A, Vicencio JM, Soussi T and Kroemer G: Mutant p53 protein localized in the cytoplasm inhibits autophagy. Cell Cycle 7: 3056-3061, 2008.

21. Vaqué JP, Fernández-García B, García-Sanz P, Ferrandiz N, Bretones G, Calvo F, Crespo P, Marín MC and León J: c-Myc inhibits Ras-mediated differentiation of pheochromocytoma cells by blocking c-Jun up-regulation. Mol Cancer Res 6: 325-339, 2008. 
22. Watanabe H, Tokuyama H, Ohta H, Satomura Y, Okai T, Ooi A, Mai M and Sawabu N: Expression of placental alkaline phosphatase in gastric and colorectal cancers. An immunohistochemical study using the prepared monoclonal antibody. Cancer 66: 2575-2582, 1990 .

23. van Dieck J, Teufel DP, Jaulent AM, Fernandez-Fernandez MR, Rutherford TJ, Wyslouch-Cieszynska A and Fersht AR: Posttranslational modifications affect the interaction of S100 proteins with tumor suppressor p53. J Mol Biol 394: 922-930, 2009.

24. van Dieck J, Fernandez-Fernandez MR, Veprintsev DB and Fersht AR: Modulation of the oligomerization state of p53 by differential binding of proteins of the S100 family to p53 monomers and tetramers. J Biol Chem 284: 13804-13811, 2009.

25. Fernandez-Fernandez MR, Veprintsev DB and Fersht AR: Proteins of the S100 family regulate the oligomerization of p53 tumor suppressor. Proc Natl Acad Sci USA 102: 4735-4740, 2005.

26. Orre LM, Panizza E, Kaminskyy VO, Vernet E, Gräslund T, Zhivotovsky B and Lehtiö J: S100A4 interacts with p53 in the nucleus and promotes p53 degradation. Oncogene 32: 5531-5540, 2013.
27. Berge G, Costea DE, Berg M, Rasmussen H, Grotterød I, Lothe RA, Mælandsmo GM and Flatmark K: Coexpression and nuclear colocalization of metastasis-promoting protein S100A4 and p53 without mutual regulation in colorectal carcinoma. Amino Acids 41: 875-884, 2011.

28. Scian MJ, Stagliano KE, Anderson MA, Hassan S, Bowman M, Miles MF, Deb SP and Deb S: Tumor-derived p53 mutants induce NF-kappaB2 gene expression. Mol Cell Biol 25: 10097-10110, 2005.

29. Zalcenstein A, Stambolsky P, Weisz L, Müller M, Wallach D, Goncharov TM, Krammer PH, Rotter V and Oren M: Mutant p53 gain of function: Repression of CD95 (Fas/APO-1) gene expression by tumor-associated p53 mutants. Oncogene 22: 5667-5676, 2003.

30. Rosty C, Ueki T, Argani P, Jansen M, Yeo CJ, Cameron JL, Hruban RH and Goggins M: Overexpression of S100A4 in pancreatic ductal adenocarcinomas is associated with poor differentiation and DNA hypomethylation. Am J Pathol 160: 45-50, 2002. 